\title{
COVID-19: extraño nuevo virus, endotelio y enseñanzas de una pandemia La incertidumbre genera miedos
}

\author{
COVID-19: strange new virus, endothelium and \\ lessons learned from a pandemic \\ Uncertainty breeds fears
}

EnRique Melgarejo-Rojas • Bogotá, D.C. (Colombia)

DOI: https://doi.org/10.36104/amc.2020.1918

La pandemia COVID-19 sorprendió a la humanidad a través de un virus desconocido y con un comportamiento "extraño": para lo cual obviamente no estábamos preparados y que por sus características después de dos meses tornó a epidemia, y tan solo un mes después en pandemia y podrá mutar finalmente a endemia. Hasta la fecha (13 agosto) ha generado 758761 muertos con una tasa de mortalidad promedio de $3.6 \%$ y casos totales confirmados de 21079074 . En Colombia hay reportados a la fecha 433805 confirmados, 14451 fallecidos con una mortalidad de $3.26 \%$.

Se inició como tal, aparentemente el 31 de diciembre de 2019, cuando un grupo de casos de neumonía de origen desconocido se informó en Wuhan, provincia de Hubei, China. El 9 de enero de 2020, el Centro para el Control y la Prevención de Enfermedades de China informó que el agente causante es un nuevo coronavirus 2 del síndrome respiratorio agudo grave (SARS-CoV-2). La enfermedad causada por SARS-CoV-2 fue posteriormente denominada COVID-19 por la OMS $(2,3)$.

Como ya es sabido, este virus utiliza la enzima convertidora de angiotensina 2 (ACE2) del sistema renina-angiotensina como receptor de entrada inicialmente en las células epiteliales pulmonares alveolares TIPO II (incluyendo cilias) $(4,5)$.

Puede manifestarse como un simple "catarro" o gripa sin consecuencias mayores, pero en algunos casos puede presentarse una desregulación del sistema inmune asociado con alteración endotelial -en realidad una endotelitis e incluso una sepsis de origen viral-con una respuesta inflamatoria, inmunológica y protrombótica exagerada con manifestaciones sistémicas, pudiendo llegar finalmente a una falla multisistémica y a la muerte. Esto ha sido llamada "tormenta de citokinas", debido a la sobreexpresión de estas polimorfas y complejas moléculas desencadenando un incremento inusitado a nivel sistémico: (IL)-2, IL-6, IL-7, del factor estimulante de colonias de granulocitos, del C-X-C motif chemokine 10e (CXCL10), de la chemokina (C-C motif), del ligando 2 (CCL2), y del factor de necrosis tumoral- alfa (TNF $\alpha$ ), interferón C , además de la ferritina, agravando esta última la oxigenación tisular y celular tempranamente (6-8).

Algunas de estas citokinas están involucradas también con la enfermedad hipertensiva, de ahí que la hipertensión arterial (HTA) no controlada o no tratada como comorbilidad, es un marcador de mayor mortalidad en enfermos COVID-19 (9). La linfopenia es un hallazgo frecuente y precoz, y recientemente se ha demostrado que hay alguna correlación entre severidad o cronicidad de la HTA y linfocitos (9-11) produciéndose mayor elevación de citokinas proinflamatorias en hipertensos no controlados, especialmente si están linfopénicos. Es decir, hay una correlación de coincidencia entre la respuesta inflamatoria del COVID-19 con la enfermedad hipertensiva (que al fin de cuentas tiene un comportamiento inflamatorio a nivel vascular, incluyendo rigidez arterial y alteraciones biomecánicas) $(12,13)$.
Dr. Enrique Melgarejo-Rojas: FACC, FESC. Profesor Emérito Hospital Militar Central. Coordinador Grupo de Trabajo Medicina Vascular, Sociedad Colombiana de Cardiología. Bogotá, D.C. (Colombia).

Correspondencia: Dr. Enrique Melgarejo-Rojas. Bogotá, D.C. (Colombia).

E.mail: enrique.melgarejo@gmail.com Recibido: 16/VI/2020 Aceptado: 23/VII/2020 
Dicho lo anterior, no es sorprendente que la diabetes mellitus-2 (DM-2) (que también ha demostrado ser una comorbilidad de alto impacto), y teniendo en cuenta que la DM-2 es una enfermedad asociada a un estado inflamatorio-oxidativo con disfunción endotelial precoz y acelerada. Igual sucede con la falla cardiaca (inflamación vascular, rigidez arterial y oxidación), y en la obesidad, en la cual el adipocito es un magnificador de la AgII con su consecuente propensión y facilitación a inflamación y oxidación (14-16).

Hubo inicialmente una controversia por confusión de que el bloqueo del sistema renina-angiotensina por antihipertensivos tipo IECAS o BRAs podrían magnificar el daño o el riesgo para COVID-19 al sobreexpresarse el receptor ECA-2 por estos medicamentos, pero esta enzima es diferente estructural y biológicamente a la ECA circulante y tisular. La ECA-2 es un contrarregulador de la AgII, convirtiéndola en Ag 1-7, la cual a través de su acción sobre los receptores Mas, genera una respuesta totalmente antagónica a la "mala" angiotensina II, incluyendo neutralización de su efecto inflamatorio, estimulando además la producción del óxido nítrico (NO) con todas sus bondades vasculares conocidas (antiinflamatorias, antiproliferativas, antifibróticas, antioxidantes, antitrombóticas, profibrinolíticas, antiaterogénicas, etc.). El SARS-CoV-2 al internalizarse en la célula una vez ligado al receptor, con la ayuda del ADAM17 (17) (que es una proteína transmembrana disintegrina y metaloproteasa (18), la cual es activada por la proteína $S$ viral, cliva a la ECA2 produciendo su eliminación de la superficie celular al penetrar a la célula, permitiendo de esta manera que la AgII haga todo el daño sin tener quien la contrarregule; es aquí cuando se inicia el verdadero daño. Pero el ADAM-17 convierte la ECA-2 en circulante y ésta no está influenciada por los IECAs o BRAs.

Y empeorando la situación, la reducción de la ECA2 pulmonar activa el receptor B1 de la bradikinina (BKDR1R) generando mayor inflamación y daño vascular no solo pulmonar sino en otros órganos (a través de la proteína $\mathrm{G}$ estimuladora). Esto demuestra la interrelación entre el sistema kinina-kalicreína (KKS) y el sistema renina-angiotensina (SRA) (17).

Por otra parte, estudios epidemiológicos han demostrado que los enfermos hipertensos que toman inhibidores del sistema renina-angiotensina (ISRA) (IECAs, BRAs) tienen menor incidencia de neumonía adquirida en la comunidad, lo cual desmitifica el riesgo del uso de estos inhibidores frente a la COVID-19 (18).

También recientemente hay publicaciones en los cuales no se demuestra que exista aumento de mortalidad con el empleo de los ISRA en pacientes infectados, aclarándose el infundado temor de que los inhibidores de este sistema, al sobre expresar los receptores ECA-2, facilitarían el daño o la letalidad del SARS-CoV-2, algo lejos de la plausibilidad y de la realidad. Al respecto, la data muestra que en una serie de 1128 hipertensos con COVID-19 de un estudio retrospectivo multicéntrico hubo menor mortalidad en pacientes que recibían ISRA (3.7\% vs. 9.8\%; P = 0.01) (19).
Otro análisis de un registro de 8910 pacientes tampoco demostró aumento de la mortalidad con los ISRA (20). Además, el sistema RA también está vinculado al sistema de péptidos natriuréticos, otro sistema contrarregulador que estimula la diuresis, la natriuresis y la vasodilatación. Los péptidos natriuréticos son inactivados por la neprilisina (endopeptidasa neutral NEP), cuya inhibición mediante el sacubitril logra activar la vía MasR mediante la generación de Ang1-7 a partir de la AngII (acción similar a la ECA-2). Es lo que conocemos hoy como ARNIs (inhibidores de la neprilisina del receptor de angiotensina). Como es de amplio conocimiento, el sacubitril en combinación con un bloqueador de receptor de angiotensina II ha denotado un punto de inflexión para el manejo de la falla cardiaca, incluso ya reconocido por las diferentes guías para el manejo de la falla cardiaca (2017) ACC/AHA.ACCF (21).

Ya se mencionó que la ACE2 se expresa en el pulmón (células epiteliales alveolares y otros tejidos incluyendo el cerebro, riñón, tracto gastrointestinal, tejido adiposo, testículos, cardiomiocitos, y variablemente en los vasos sanguíneos (células endoteliales y células musculares lisasvasculares). Es decir, involucra la pared vascular y su templo: el endotelio. Este daño endotelial por el SARS-CoV-2 y su respuesta inflamatoria, puede desestabilizar placas ateroscleróticas vulnerables, inducir además inflamación y/o citotoxicidad cardiaca y activación de la cascada de coagulación llevando a un estado de hipercoagulación conduciendo finalmente hacia una coagulación intravascular diseminada $(22,23)$. Estudios en autopsia (que inicialmente fueron pocas), se documentó que la COVID-19 no era una "simple neumonía viral", como ya fue mencionado anteriormente, había compromiso micro y macrotrombótico partiendo del daño endotelial y vascular con un componente microtombótico inicial, lo cual explica la hipoxemia en enfermos aun asintomáticos; alteración de la angiogénesis posteriormente y expresión de biomarcadores de lesión cardiaca como manifestaciones de depresión de la función ventricular y arritmias (empeoradas por cloroquina, hidroxicloroquina, azitromicina y demás fármacos que prolongan el intervalo QT del electrocardiograma), aunadas por el desbalance hidroelectrolítico (especialmente hipokalemia y/o hipomagnasemia). Hay manifestaciones de cardiotoxicidad directa (24-26), incluyendo elevación de troponinas. Esta cardiotoxicidad se ha demostrado mediante resonancia magnética y ecocardiografía, documentándose un cuadro de cardiomiopatía por estrés cardíaco agudo (Takotsubo) (27).

Por otro lado, la inmovilización prolongada, aunada a la disfunción endotelial, inflamación y estado protrombótico, facilitan también trombosis venosa profunda y/o embolia pulmonar demostradas clínicamente por el aumento del dímero D y del fibrinógeno. El hallazgo de estos dos marcadores elevados indican ya un mal pronóstico (28).

Ante la evidencia que también ha fallecido gente joven sin aparentes comorbilidades, cabe la posibilidad que la disfunción endotelial en la etapa subclínica sea la explicación. Posiblemente además de los biomarcadores e image- 
nología, evaluar función endotelial o detectar su disfunción no invasiva en la población joven infectada, pueda ser una manera de pronosticar el riesgo de complicaciones en este grupo poblacional. Este es un tópico que deberá tenerse en cuenta, el tiempo lo dirá.

En resumen, la pandemia COVID-19 a nivel del sistema vascular en la población vulnerable genera disfunción endotelial precoz, progresiva y severa, aunque afortunadamente es un porcentaje bajo (según diferentes reportes entre un 3-7\%). Genera concomitantemente hipoxemia temprana, tormenta de citoquinas, cardiotoxicidad y coagulación intravascular diseminada, manifestándose clínicamente como falla cardíaca, infartos en general, arritmias y tromoboembolismo de origen venoso, falla multisitémica y muerte.

A nivel específico cardiaco se ha reportado en diferentes series falla cardiaca, arritmias ventriculares malignas (torsades de pointes, taquicardia ventricular y fibrilación ventricular), falla cardiaca, shock cardiogénico, miocarditis y cor pulmonale agudo o TEP. No eran de esperarse tantas complicaciones cardiovasculares-sistémicas para un coronavirus, y por lo tanto, no hemos aun aprendido a manejarlo, ya que tiene diferentes etapas de severidad -desde los leves síntomas- hasta el colapso cardiovascular y sistémico.

\section{Conclusiones y enseñanzas}

Nos llegó un virus nuevo y atípico, entre otras, por su altísima capacidad de propagación y de diseminación, incluso, en algo atípico: su diseminación en la etapa asintomática. Aunque su letalidad no es muy alta comparativamente con el SARS-2 y el MERS (producidos también por coronavirus), y el ébola y otras epidemias como dengue, chikungunya, etc., ha causado mayor mortalidad que el SARS y el MERS (29), debido a su rápida difusión y como se mencionó, transmitirse en etapa asintomática y la ausencia de tratamiento específico, mucho desconocimiento y no pedagogía de impacto hacia la comunidad que por ignorancia no tiene la cultura del autoconfinamiento, y peor aun, del autocuidado. Pero el impacto en los sistemas de salud -incluyendo saturación de camas UCI-, más el impacto socioeconómico mundial y geopolítico, aunado a la carencia, aún, de un tratamiento específico y la demora lógica de la vacuna (porque para complicar más las cosas, es un virus mutante que dificultará su tipificación de la cepa(s) más patógena(s) y por lo tanto todo parece indicar que el SARS-CoV-2 llegó para quedarse endémico al igual que la influenza y el VIH, es decir que pasaremos de la pandemia a la endemia. Y que lo que se pensaba inicialmente que el manejo era como el de una neumonía tipo SARS, los ventiladores no han sido la solución. Someter a un pulmón inflamado y microtrombosado a carga de presión y volumen, ha demostrado que no es la solución mágica ni racional y así lo demuestran las estadísticas $(30,31)$.

Y quizás, el problema más grave al cual tenemos que enfrentarnos, es al miedo, el cual paraliza nuestro comportamiento y puede ser incluso más grave que la pandemia, y también el síndrome post-COVI o el tsunami coronavirus; es decir, sus secuelas y posibles réplicas (como fibrosis pulmonar residual en los recuperados, estado post-embolia pulmonar, síndrome post-UCI prolongado más el estrés posttraumático, asociado a ansiedad-depresión, falla cardiaca residual o empeorada, fracturas, convalecencia prolongada, etc.). Aunados con problemas socio-económicos planetarios, geopolíticos por aumento de la pobreza, disparo del desempleo, desnutrición, sarcopenia severa en los ancianos excesivamente e indiscriminadamente confinados, osteoporosis, muertes en la casa por complicaciones de enfermedades no COVI, y quizás un fenómeno no visto antes: desbordamiento de fallas cardiacas, diabetes descontroladas, HTA no controladas, enfermedad coronaria no intervenida, arritmias más avanzadas, entre otros, que saldrán masivamente a los hospitales, porque los enfermos se contuvieron para acudir oportunamente para ayuda hospitalaria por el "miedo a que me peguen el virus en el hospital" y el aplazamiento de intervencionismo y cirugías "no urgentes", explosionará y también desbordará significativamente el sistema de salud. Estamos ante una pandemia rápidamente diseminada (globalización, aeropuertos y aviones que llevan la COVID a más de $900 \mathrm{Km} / \mathrm{h}$, aunada al hecho de no estar preparados en cuanto a talento humano y tecnológico ni toma masiva de muestras; pero, no simplemente ni como "estadística diaria", sino tomar medidas de cerco epidemiológico, y no encerrando a los sanos y a los enfermos por decisiones políticas. Además, las evidentes falencias en los sistemas de salud nacionales especialmente sus precariedades regionales por abandono del concepto de salud; por el crónico destrabajo de los políticos, y tristemente agravado por la otra epidemia-endemia nacional: la corrupción que quizás es más grave y que ha sido crónicamente a través del tiempo más letal que la misma COVID-19. Una actitud inhumana que cuesta trabajo entenderla.

Sólo nos queda actuar con lo evidente por ahora: las medidas más efectivas y menos costosas pero muy duras emocionalmente como el distanciamiento social para evitar una alta carga viral al receptor ACE2 tal y como nos lo demuestra un metaanálisis publicado recientemente en Lancet (32). El uso adecuado del tapabocas, evitar tocarse la cara y los ojos, y la distancia entre personas a más de un metro resultan ser las medidas más eficaces. Las muestras de afecto con un apretón de manos, abrazos y besos, nos tocó aplazarlos, nadie sabe hasta cuándo. Pero mientras que tengamos palabras para manifestar nuestro afecto es el cambio racional. $Y$ un punto crucial, es educar $y$ concientizar a la gente, que ésta no es una "gripita fuerte" más. No, es una enfermedad desconocida que llegó sin saber cómo, y llegó para quedarse un tiempo desconocido y con rebrotes en pacientes ya infectados. Han cambiado muchas de nuestras costumbres, pero con la esperanza que de pronto cambiemos nosotros mismos o nos autodescubramos en la soledad del confinamiento y replantear nuestra actitud solidaria ante los demás y hacia la madre naturaleza, cuidando nuestra casa: el 
planeta tierra, que de alguna manera se puede estar manifestando ante la depredación del Antropozeno. Puede ser esta una única o última oportunidad, que de pronto no volverá.

En la práctica una estructura ultramicroscópica nos ha cambiado "la normalidad". En la teoría, sería esperanzador que del asteroide B612 volviese El Principito a comentarnos amablemente sobre la forma tan extraña como los adultos vemos la vida. Pero nuestra estulticia y la condición humana que de tanto habló Anna Arendt, nos deja vislumbrar un no volver a lo "normal". Volveremos seguramente a vivir de una manera diferente conviviendo con un virus más mientras que la ciencia (ahora más valorada) logre contenerlo y los terrícolas, ojalá hayamos aprendido algo del comportamiento de otras especies: unirse ante las amenazas, en nuestro caso, ante la incertidumbre.

\section{Referencias}

1. WHO. Disponible: App Covid 19 WHO, Worldometers, info, JHU -Apple-store, Android

2. Zhou P, Yang X-L, Wang X-G, Hu B, Zhang L, Zhang W, Si H-R, Zhu Y, Li B, Huang C-L, Chen H-D, Chen J, Luo Y, Guo H, Jiang R-D, Liu M-Q, Chen Y, Shen X-R, Wang X, Zheng X-S, Zhao K, Chen Q-J, Deng F, Liu L-L, Yan B, Zhan F-X, Wang Y-Y, Xiao, G-F, Shi Z-L. A pneumonia outbreak associated with a new coronavirus of probable, bat origin. Nature 2020;579:270-273.

3. http://www.euro.who.int/en/health-topics/health-emergencies/coronaviruscovid-19/ news/news/2020/3/who-announces-covid-19-outbreak-a-pandemic. (30 March 2020)

4. Hoffman M, Klein-weber, Schroeder s et al. SARS-Cov-2 Cell entry Depends on ACE2 and TMPRSS2 and is Blocked by a Clinically Proven Protease.(2020) Cell 181,52; 271/280

5. Hirano T, Murakami M. 2020. Inmunity 52,731/733.COVID-19: a New Virus, but a Familiar Receptor and Cytokine Release Syndrome.

6. Wong CK, Lam CWK, Wu AKL, et al. Plasma inflammatory cytokines and chemokines in severe acute respiratory syndrome. Clin Exp Immunol 2004;136 (1):95-103 2020. ACE Inhibitors and ARBs: Good, Bad, or Neutral in COVID-19 Patients?

7. Ruan Q, Yang K, Wang W, et al. Clinical predictors of mortality due to COVID-19 based on an analysis of data of 150 patients from Wuhan, China. 2020 Intensive Care Med 46, 846, 848 .

8. Klok FA, Kruip MJHA, van der Meer NJM, et al. Incidence of thrombotic complications in critically ill ICU patients with COVID-19. 2020. Thromb Res $.191: 145-147$

9. Zhou F, Yu T, Du R, et al. Clinical course and risk factors for mortality of adult inpatients with COVID-19 in Wuhan, China: a retrospective cohort study [published correction appears in Lancet. 2020 Mar 28;395(10229):1038] [published correction appears in Lancet. 2020 Mar

10. Siedlinski M, Jozefczuk E, Xu X, Teumer A, Evangelou E, Schnabel RB, Welsh P,Maffia P, Erdmann J, Tomaszewski M, Caulfield MJ, Sattar N, Holmes MV, Guzik TJ. White blood cells and blood pressure: a Mendelian randomization study. 2020, Circulation .119 .045102 .

11. M, Mikolajczyk T, Sliwa T, Dikalov S, Weyand CM, Guzik TJ, Harrison DG. Hypertension and increased endothelial mechanical stretch promote monocyte differentiation and activation: roles of STAT3, interleukin 6 and hydrogen peroxide. Cardiovasc Res 2018; 114: 1547-1563.

12. Gavin Y Oudit ; Marc A Pfeffer. Plasma angiotensin-converting enzyme 2: novel biomarker in heart failure with implications for COVID-European Heart J. Volume 41 Issue 19. 14 May 2020. https://doi.org/10.1093/eurheartj/ehaa414.
13. Mitchell GF, Guo CY, Benjamin EJ, et al. - Cross-sectional correlates of increased aortic stiff ness in the community: the Framingham Heart Study. Circulation. 2007; 115: 2628-2636.

14. Avogrp A, Albiero M, Mangazzo L et al. Diabetes Care 2011, Diabetes Meelitus-2 \}: an inflamatory state. 34 Suppl 2 ,S285-290.

15. Drexler H.Endothelium as Thrapeutic Target in Hert Failure. Circulation (1999) 98, 2252-5.

16. Ellulu MS, Patimah I, Khaza'ai H, Rahmat A, Abed Y. Obesity and inflammation: the linking mechanism and the complications. Arch Med Sci. 2017;13(4):851863. doi:10.5114/aoms.2016.58928

17. Kreutz R, Algharably EAE, Azizi M, et al. Hypertension, the renin-angiotensin system, and the risk of lower respiratory tract infections and lung injury: implications for COVID-19.Cardiovasc Res. 2020;116(10):1688-1699. doi:10.1093/cvr/ cvaa097

18. Janssen DJA, Ekström M, Currow DC, et al. COVID-19: Guidance on Palliative care from a European Respiratory Society International Task Force [published online ahead of print, 2020 Jul 16]. Eur Respir J. 2020;2002583. doi:10.1183/13993003.02583-2020

19. Wu A, Good C, Downs JR, Fine MJ, Pugh MJ, Anzueto A, Mortensen EM. The association of cardioprotective medications with pneumonia-related outcomes. PLoS One 2014;9:e85797

20. Rohit L, Liu PP and Li H. Association of Inpatient Use of Angiotensin Converting Enzyme Inhibitors and Angiotensin II Receptor Blockers with Mortality Among Patients With Hypertension Hospitalized With COVID-19. Circ Res. 2020, 10.1161/CIRCRESAHA.120.317134

21. Yancy CW, Jessup M, Bozkurt B, et al. 2017 ACC/AHA/HFSA Focused Update of the 2013 ACCF/AHA Guideline for the Management of Heart Failure: A Report of the American College of Cardiology/American Heart Association Task Force on Clinical Practice Guidelines and the Heart Failure Society of America. Circulation. 2017;136(6):e137-e161. doi:10.1161/CIR.0000000000000509

22. Liang Chen1, Xiangjie Li , Mingquan Chen, Yi Feng Chenglong Xiong. The ACE2 expression in human heart indicates new potential mechanism of heart injury among patients infected with SARS-CoV-19. Cardiovascular Research (2020) 116, 1097-1100.

23. Levi M, van der Poll T, Büller HR. Bidirectional relation between inflammation and coagulation. Circulation 2004;109:2698-704.

24. Prabhu SD. Cytokine-induced modulation of cardiac function. Circ Res. 2004;95 (12):1140-53

25. Zhou F, Yu T, Du R, et al. Clinical course and risk factors for mortality of adult inpatients with COVID-19 in Wuhan, China: a retrospective cohort study. Lancet 2020; 395: 1054-62.

26. Zheng YY, Ma YT, Zhang JY, Xie X. COVID-19 and the cardiovascular system. Nat Rev Cardiol. 2020;17(5):259-260. doi:10.1038/s41569-020-0360-5

27. Sala S, Peretto G, Gramegna M, et al. Acute myocarditis presenting as a reverse TakoTsubo syndrome in a patient with SARS-CoV-2 respiratory infection. 2020 Eur Heart J 2020. doi:10.1093/eurheartj/ehaa286.

28. Meizlish M, Pine A, Goshua G, et al. Circulating Markers of Angiogenesis and Endotheliopathy in COVID-19. Preprint. medRxiv. 2020;2020.06.29.20140376. Published 2020 Jul 1. doi:10.1101/2020.06.29.20140376

29. Mahase E. Coronavirus covid-19 has killed more people than SARS and MERS combined, despite lower case fatality rate. BMJ. 2020;368:m641. Published 2020 Feb 18. doi:10.1136/bmj.m641

30. Marini JJ, Rocco PRM, Gattinoni L. Static and Dynamic Contributors to Ventilator-induced Lung Injury in Clinical Practice. Pressure, Energy, and Power. Am J Respir Crit Care Med.2020;201(7):767-774. doi:10.1164/rccm.201908-1545C

31. Patel BK, Kress JP, Hall JB. Alternatives to Invasive Ventilation in the COVID-19 Pandemic [published online ahead of print, 2020 Jun 4]. JAMA. 2020;10.1001/ jama.2020.9611. doi:10.1001/jama.2020.9611

32. https://www.acc.org/latest-in-cardiology/journal-scans/2020/06/08/12/37/physical-distancing-face-masks-and-eye-protection?utm_source=accupdate\&utm medium=email_newsletter\&utm_content=20200612\&utm_campaign=accupdate 